A. Weisbecker, M. Burmester \& A. Schmidt (Hrsg.): Mensch und Computer 2015 Workshopband, Stuttgart: Oldenbourg Wissenschaftsverlag, 2015, S. 45-50.

\title{
Entwicklung eines HCD-Leitfadens für Krisenmanagementsysteme
}

\author{
Daniel Orlowski, Johannes Sautter
}

Fraunhofer-Institut für Arbeitswirtschaft und Organisation IAO

\section{Zusammenfassung}

Krisenmanagementsysteme werden sich nur dann flächendeckend durchsetzen können, wenn die Mensch-Computer-Interaktion im Krisenszenario reibungslos funktioniert. Die Berücksichtigung von Usability-Anforderungen sowohl bei der Planung und Entwicklung, als auch bei der Evaluation der Systeme ist daher von besonderer Bedeutung. Leider fehlen den verschiedenen Interessensvertretern verbindliche Standards und konkrete Hilfestellungen, wie Human Centered Design (HCD) in Hinblick auf Krisenmanagementsysteme anwendbar ist. Dieser Artikel beschreibt die geplante Entwicklung eines Leitfadens zur Auswahl und Durchführung von HCD-Methoden bei Krisenmanagementsystemen, insbesondere als abschließende Evaluation solcher Systeme.

\section{$1 \quad$ Einleitung}

Krisen sind Naturkatastrophen, technische Fehlfunktionen oder Unfälle, die ernste Folgen für Mensch und Natur haben, wenn nicht frühzeitig, angemessen darauf reagiert wird. Einsatzkräfte stehen in Krisensituationen unter hohem psychischem und physischem Druck. Insbesondere ist dies in der Chaosphase der Fall, wenn sie sich beispielswiese bei einem Massenfanfall von Verletzten (MANV) mit vielen Schwerverletzte konfrontiert sehen, ohne die komplette Lage überblicken zu können. Dennoch sind sie aufgrund akuter Gefährdung der Schwerverletzten gezwungen, schnelle Entscheidungen zu treffen (Mentler 2011, Karsten 2014).

Interaktive Systeme in diesem Bereich, so genannte Krisenmanagementsysteme, können zur Planung, Training und zur Bewältigung der Krise genutzt werden. Sie haben das Potential die Arbeit der Einsatzkräfte entscheidend zu verbessern. In der Krisensituation vereinfachen sie den Informationsaustausch unterhalb der Akteure und bieten ihnen Entscheidungshilfen.

Obwohl das große Potenzial von Krisenmanagementsystemen schon seit Jahren erkannt ist (z.B. Lindgaard et al. 2010; Gao et al. 2007), gibt es noch immer keine flächendeckende 
Nutzung der Systeme durch Behörden und Organisationen mit Sicherheitsaufgaben in Deutschland (BOS) (Mentler \& Herczeg 2014).

Bestehende Forschung verweist vor diesem Hintergrund eindringlich auf die Wichtigkeit von Usability als ein zentrales Qualitätsmerkmal bei Krisenmanagementsystemen: Nicht die Technologie ist die größte Hürde der Entwicklung solcher Systeme, sondern deren Gebrauchstauglichkeit für Einsatzkräfte in der Krise (Nestler 2014).

In der Forschung herrscht Einigkeit darüber, dass eine hohe Gebrauchstauglichkeit interaktiver Systeme nur durch den frühzeitigen und kontinuierlichen Fokus auf Nutzer und deren Aufgaben in der Entwicklung der Systeme, erreicht werden kann (Gould \& Lewis 1985, Norman \& Draper 1986, Shneiderman 2010). Dennoch gibt es, in Bezug auf Krisenmanagementsysteme, weder passende Hilfestellung noch verbindliche Standards, wie eine solche menschzentrierte Entwicklung aussehen soll.

Der vorliegende Artikel skizziert das Vorhaben einen Human Centered Design-Leitfaden zur Anwendung bei Krisenmanagementsystemen zu konzipieren. Der Leitfaden stellt HCDMethoden vor, um Prototypen und fertige Krisenmanagementsysteme zu evaluieren. Diese Evaluation kann von BOS genutzt werden um festzustellen, ob die Anforderungen der Nutzer an das entsprechende System erfüllt werden. Gleichzeitig erlaubt es die Evaluation, verschiedene Systeme miteinander zu vergleichen.

Der Leitfaden soll dabei BOS die Vorteile von HCD bei Krisenmanagementsystemen näherbringen. Außerdem werden Rollen und Aufgaben einzelner Projektbeteiligter bei der Evaluation der Systeme vorgeschlagen. Usability-Experten erhalten darüber hinaus Informationen wie eine erweiterte Usability-Evaluation eines Krisenmanagementsystems aussehen kann.

\section{Stand der Forschung}

Während der Krise müssen Krisenmanagementsysteme effizient, einfach und fehlerfrei bedienbar sein. Anwender haben keine Zeit sich mit der Bedienung auseinanderzusetzen. „Bereits kleinere Fehler im User Interface führen zum Scheitern der Mensch-ComputerInteraktion (MCI). Scheitert in dem Krisenszenario die Interaktion, scheitert das System" (Nestler 2014).

In verschiedenen, existierenden Forschungsprojekten begegnen Usability-Experten diesen hohen Usability-Anforderungen mit Hilfe von verschiedener HCD-Prozessen (z.B. Sautter et al. 2012; Kluckner et al. 2014). Kindsmüller et al. (2011) schlagen für die Entwicklung und den Einsatz eines Krisenmanagementsystems ein Modell namens „Care \& Prepare“ vor. Kern des Ansatzes ist es, das Krisenmanagementsystem als eine Erweiterung eines bestehenden, im beruflichen Alltag genutzten Einsatzsystems zu entwickeln. Dadurch würden Einsatzkräfte im Umgang mit dem Krisenmanagementsystem durch tägliche Anwendung geschult. In der Entwicklung der Krisenmanagementsysteme soll ein frühzeitiger und kontinuierlicher Fokus auf Nutzer und deren Aufgaben und ein iteratives Vorgehen hohe Usability 
gewährleisten. Im Zuge des Entwicklungsprozesses sollen verschiedene HCD-Methoden, wie beispielsweise Interviews, Usability Tests und Feldstudien, zum Einsatz kommen.

Nestler (2014) betont in diesem Zusammenhang, dass klassische Usability-Tests in Krisensimulationen unumgänglich sind, um verlässliche Aussagen über die Nutzbarkeit der Systeme treffen zu können. Er merkt allerdings an, dass ein Usability-Test, welcher Eigenschaften einer realen Krise hat, ebenso komplex wie ressourcenfressend ist (Nestler 2014; Mentler et al. 2011).

Obwohl die Forschung die Bedeutung von Usability betont und beispielhaft aufzeigt wie ein Entwicklungsvorgang mit Hilfe von HCD aussieht, gibt sie wenig konkrete Unterstützung bei der Auswahl der Methoden bezogen auf Krisenmanagementsysteme.

Erschwerend kommt hinzu, dass die Anwendung menschzentrierter Methoden bei Krisenmanagementsystemen selbst für Usability-Experten überaus anspruchsvoll ist (Chilana et al. 2010, Nestler 2014). Es ist nicht möglich den Krisenkontext zeitlich oder räumlich einzugrenzen oder vorauszusehen (Mentler \& Herczeg 2014). Zudem ist es überaus anspruchsvoll, Aussagen über konkrete Anwender zu machen. Anders als beispielsweise im Bereich der Medizintechnik gibt es keinerlei verbindlichen Standards welche einen Usability Engineering-Prozess definieren. Hinderlich ist außerdem, dass Möglichkeiten der gezielten Forderung einer Begleitung nach Ergonomie-Gesichtspunkten bei BOS, die als Auftraggeber fungieren, sowie die Zuständigkeiten verschiedener Stakeholder in der Entwicklung und Wartung von Krisenmanagementsystemen in Bezug auf Usability noch nicht hinreichend besprochen wurden.

\section{Forschungsansatz und Vorgehen}

Die vorliegende Arbeit postuliert einen HCD-Leitfaden, als eine Hilfestellung bei der Auswahl und Anwendung von HCD-Methoden bei Krisenmanagementsystemen. Der Leitfaden soll dabei zwei Ausführungen haben. Die Kurzfassung soll BOS die Relevanz von HCD nahebringen und die strukturelle Planung von Usability-Engineering erläutern. Es finden sich Erklärungen darüber, warum HCD in diesem Bereich besonders anspruchsvoll ist. Außerdem wird eine Auswahl von drei Methoden vorgestellt, welche sich zur Anwendung bei Krisenmanagement- und sicherheitskritischen Systemen bewährt haben, gleichzeitig aber zeit- und kosteneffektiv sind (Nielsen 1994, Kindsmüller et al. 2011, Zhang et al. 2003). Die in der ersten Version enthaltenen Methoden des Leitfadens sind Kontextanalyse, Think-Aloud und Heuristische Evaluation. Die erweiterte Fassung des Leitfadens richtet sich an UsabilityExperten und gibt Handlungsempfehlungen zur Wahrnehmung ihre Rolle bei Vorbereitung und Durchführung der Methoden.

Abgeleitet aus den praktischen Erfahrungen von Offergeld \& Oed (2006), beleuchtet der Leitfaden die Bedeutung und Anwendung von HCD für Organisationen welche Krisenmanagementsysteme nutzen. Es ist anzunehmen, dass HCD von IT-Unternehmen nur dann berücksichtigt wird, wenn BOS als Auftraggeber konkrete HCD-Methoden kennen und einfor- 
dern (Offergeld \& Oed 2006). Aufgrund der Schwierigkeit für Entwickler und UsabilityBerater sich in Aufgaben und Prozesse des Krisenmanagements hineinzudenken, wird außerdem vorgeschlagen, dass BOS selbst Nutzerforschung durchführen oder Usability-Beratung extern beauftragen (Chilana et al. 2010). Der Leitfaden soll somit eine Grundlage für einen Austausch zwischen BOS, Entwickler und Usability-Experten über das Thema HCD bei Krisenmanagementsystemen schaffen.

Im Folgenden wird das geplante bzw. aktuell stattfindende Vorgehen zur Erstellung des Leitfadens beschrieben: Um erste Erfahrungen mit Usability-Methoden in der Domäne zu sammeln, wurde als erster Schritt ein Usability-Test und explorative Experteninterviews durchgeführt. Mit Hilfe der Think-Aloud-Methode wurde ein Krisenmanagementsystem mit Einsatzleitern evaluiert, welches es Fachanwendern erlaubt Krisensituationen in einem BüroNutzungskontext zu Analysezwecken zu simulieren (Sautter et al. 2015). Testpersonen waren zwei Einsatzkräfte des Roten Kreuzes, die realistische Aufgaben mit Hilfe des Krisenmanagementsystems lösen sollten. Der Test gab Aufschluss über die praktische Anwendbarkeit von HCD-Methoden in diesem Bereich.

Als nächster Schritt sollen mittels Experteninterviews grundsätzliche Informationen über mögliche Zielstellungen und Eigenschaften des Leitfadens eruiert werden. Die Interviewpartner sind entweder Experten im Themenfeld Usability oder im Bereich von Krisenmanagementsystemen. Zusätzlich finden Interviews mit Personal von BOS statt, um festzustellen, ob aktuell Usability eine hinreichende Bedeutung bei der Ausschreibung und Beschaffung von Krisenmanagementsystemen findet. Die Interviews werden mit Hilfe eines Interviewleitfadens geführt. Fragestellungen sind HCD-Methoden und deren Auswahlkriterien in Bezug auf Krisenmanagementsysteme sowie Hindernisse, die es typischerweise bei der Implementierung von HCD sowohl auf Seite der Softwareentwickler, als auch auf Seite der BOS gibt.

Auf Basis der Ergebnisse der Experteninterviews soll im nächsten Schritt ein erster Entwurf des HCD-Leitfadens erstellt werden. Sowohl für die Ausführung des HCD-Leitfadens für BOS als auch für die Ausführung für Usability-Experten soll im Anschluss eine Evaluation stattfinden. Ein besonderer Fokus liegt auf der Evaluierung als Grundlage zur Auswahl mehrerer zu Verfügung stehender Systeme zur Unterstützung einer bestimmten fachlichen Fragestellung. Weiterhin soll ein Workshop mit BOS-Experten durchgeführt werden, um deren Erfahrungen mit Gebrauchstauglichkeit sowie mögliche Ansatzpunkte zur Integration für die Konzeption des Leitfadens zu identifizieren.

\section{Zusammenfassung}

Usability ist ein zentrales Qualitätsmerkmal für Krisenmanagementsysteme. Um hohe Gebrauchstauglichkeit der Systeme zu gewährleisten reicht es nicht aus von deren Entwicklern „,intuitive“ Benutzeroberflächen zu fordern. Ein Human Centered Design Leitfaden könnte helfen den unterschiedlichen Stakeholdern bei Endanwenderorganisationen, Softwareentwicklungsorganisationen und Usability-Beratern näherzubringen, wie Usability bei Krisen- 
managementsystemen realisiert werden kann und welche Zuständigkeiten sich daraus ergeben. Ziel ist BOS für die Bedeutung von Usability zu sensibilisieren und mit einem Leitfaden gezielt zu unterstützen.

\section{Danksagung}

Das Projekt DRIVER, welches durch das siebte Rahmenprogramm der Europäischen Union FP7/2007-2013 (grant agreement $n^{\circ} 607798$ ) kofinanziert wird, hat eine paneuropäische Testumgebung für Krisenmanagement-Konzepte und -systeme zum Ziel (www.driverproject.eu).

\section{Literaturverzeichnis}

Chilana, P. K., Wobbrock, J. O., \& Ko, A. J. (2010). Understanding usability practices in complex domains. In Mynatt, E.; Fitzpatrick, G.; Hudson, S.; Edwards, K.; Rodden, T. (Hrsg.) Proceedings of the SIGCHI Conference on Human Factors in Computing Systems. Atlanta, USA: ACM, S. 2337-2346.

Gao, T., Massey, T., Sarrafzadeh, M., Selavo, L., Welsh, M. (2007). Participatory User Centered Design Techniques for a Large Scale Ad-hoc Health Information System. In Kravets, R. \& Petrioli, Ch. (Hrsg.): Proceedings of the 1st ACM SIGMOBILE international workshop on Systems and networking support for healthcare and assisted living environments. New York, USA: ACM, S. 43-48.

Gould, J.D. \& Lewis, C. (1985). Designing for Usability: Key Principles and What Designers Think. In Communications of the ACM, Volume 28, Number 3.

Karsten, A. (2015). Führen durch die Chaos-Phase. In: Bevölkerungsschutz. Bonn: Bundesamt für Bevölkerungsschutz und Katastrophenhilfe

Kindsmüller, M.C., Mentler, T., Herczeg, M. \& Rumland, T. (2011). Care \& Prepare - Usability Engineering for Mass Casualty Incidents. In ACM EICS4Med 2011: Proceedings of the 1st International Workshop on Engineering Interactice Computing Systems for Medicine and Health Care, Pisa, Italy, ACM, S. 30-35.

Kluckner, S., Heintze, K., and Wendt, W. (2014) Designing for the User: Tailoring a Simulation Software Interface to the Needs of Crisis Managers, In Hiltz, S.R., Pfaff, M.S., Plotnick, L., \& Shih, P.C., (Hrsg.) Proceedings of 11 th International ISCRAM Conference, University Park, PA, USA.

Lindgaard, G., Tsuji, B., Sen, D., Lundahl, S., MacMillan, D., Anderson, M., \& Mongeau, M. (2010). Using a user - centred approach to designing a public health crisis management system. In IADIS International journal on WWW/Internet, 8(2), S. 151-166.

Mentler T. \& Herczeg M. (2014). Interactive Cognitive Artifacts for Enhancing Situation Awareness of Incident Commanders in Mass Casualty Incidents. In Stary, Ch. (Hrsg.): ECCE 2014 Proceedings of the 2014 European Conference on Cognitive Ergonomics. Vienna, Austria: ACM.

Mentler, T., Kindsmüller, M.C., Herzeg, M. \& Rumland, T. (2011). Eine benutzer- und aufgabenzentrierte Analyse zu mobilen Anwendungssystemen bei Massenanfällen von Verletzten. In Heiss, H. (Hrsg.): Informatik 2011. Bonn, Germany: Gesellschaft für Informatik.

Nestler, S., (2014). Evaluation der Mensch-Computer-Interaktion in Krisenszenarien / Evaluating human-computer-interaction in crisis scenarios. In Ziegler, J. (Hrsg.): i-com: Vol. 13, No. 1. Berlin: De Gruyter. S. 53-62. 
Norman, D. A. \& Draper, S. W. (1986). Introduction. In Norman, D. A. \& Draper, S. W. (Hrsg.): User centered system design. New perspectives on human-computer interaction. Hillsdale: Erlbaum. S. $1-6$.

Offergeld, M. \& Oed, R. (2006). Usability Engineering als Auftraggeberkompetenz. In Bosenick, T., Hassenzahl, M., Müller-Prove, M. \& Peissner, M. (Hrsg.): Proceedings Usability Professionals 2006. Stuttgart, Germany: German Chapter der Usability Professionals Association e.V.

Sautter, J., Böspflug, L. \& Schneider, F. (2015). Entwicklung eines Interaktionskonzeptes zur Simulation und Analyse von MANV-Einsätzen. In: Mensch \& Computer 2015 - Workshopband. Oldenbourg-Verlag.

Sautter, J., Engelbach, W. \& Frings, S. (2012). User-Centered Elaboration of an Integrated Crisis Management Modeling and Simulation Solution. In Aschenbruck, N.; Martini, P.; Meier, M. \& Tölle, J. (Hrsg.): 7th Security Research Conference, Future Security 2012, Berlin, Germany: Springer.

Shneiderman B., Plaisant, C. (2010). Designing the user interface: Strategies for effective humancomputer interaction, 5. Auflage. Boston, USA: Addison-Wesley. 